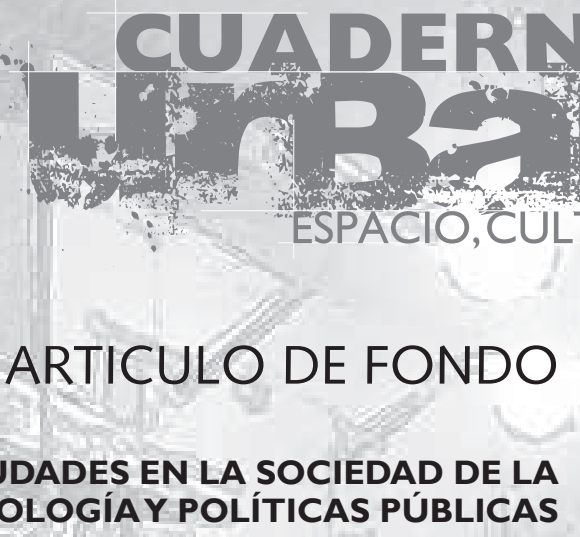
INFORMACIÓN. CIENCIA,TECNOLOGÍAY POLÍTICAS PÚBLICAS

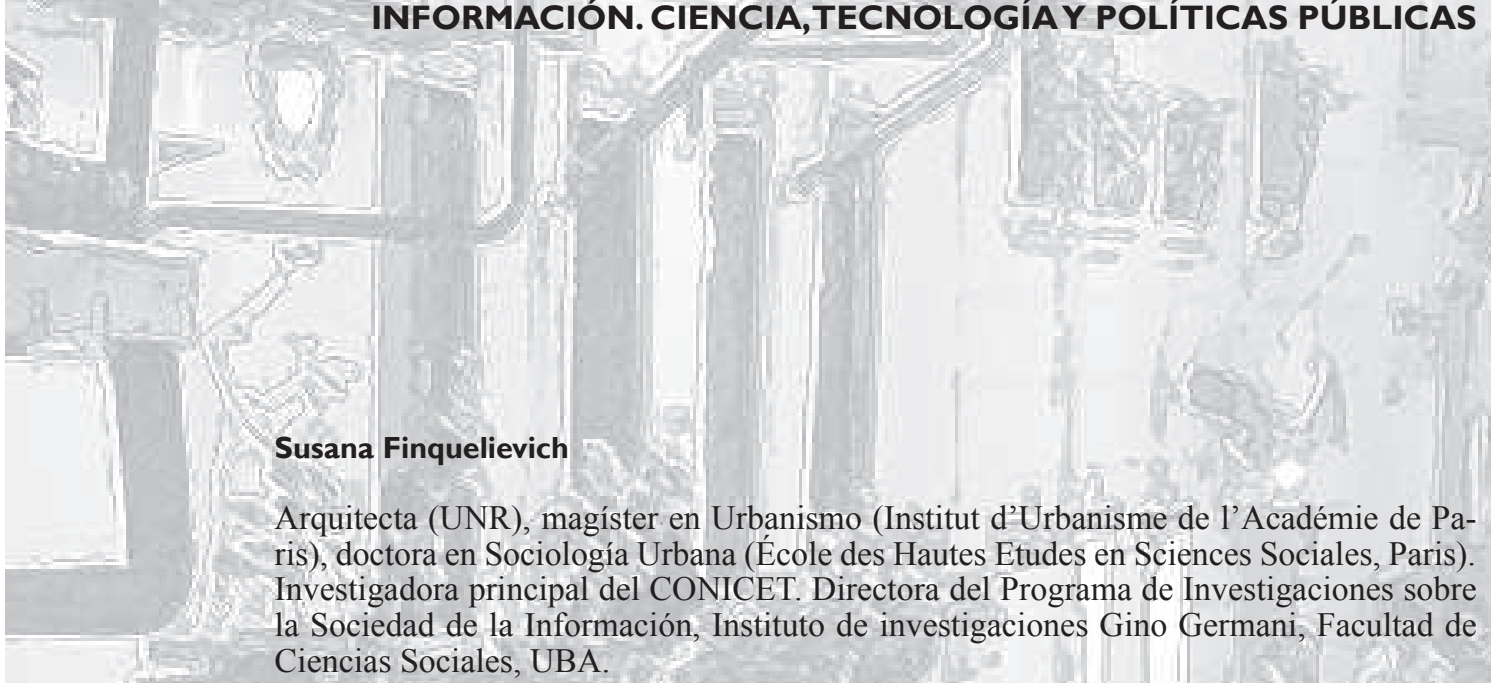

\title{
Patricio Feldman
}

Licenciado en Ciencia Política (UBA), becario doctoral del CONICET, Programa de Investigaciones sobre la Sociedad de la Información, Instituto de investigaciones Gino Germani, Facultad de Ciencias Sociales, Universidad de Buenos Aires

Instituto de investigaciones Gino Germani, Facultad de Ciencias Sociales. Universidad de Buenos Aires

ISNN 1666-6I86. Volumen 18 N. ${ }^{\circ} 18$ (Junio de 20I5) pp. 169-190 - Recibido: 22-10-I4.Aprobado: 22-II-I4 


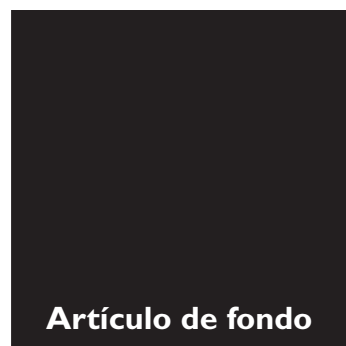

Susana Finquelievich y Patricio Feldman

\section{Resumen}

El objetivo de este trabajo es analizar las relaciones entre las ciudades y los procesos de innovación socio-tecnológica centrados en las tecnologías de información y comunicación (TIC). ¿Cómo evaluar y medir la innovación? ¿Qué metodologías emplear? Se plantean los marcos teóricos sobre los indicadores que intentan medir la producción de innovaciones en empresas, universidades, gobiernos locales y organizaciones de la sociedad civil y, fundamentalmente, las innovaciones que surgen de las interrelaciones entre estos actores sociales. Se aplican sistemas de medición de la innovación según indicadores nacionales e internacionales y se esbozan indicadores específicos para analizar, medir y comparar procesos de innovación en áreas urbanas. La metodología es cuantitativa y cualitativa. Se describen los resultados de estudios de caso en tres ciudades de diferente tamaño en la provincia de Buenos Aires, que cuentan con universidades y polos tecnológicos, y en las que se desarrollan actividades de I+D en bienes y servicios de base TIC: La Plata, Bahía Blanca y Tandil.

Palabras clave

Ciencia y tecnología, innovación socio-técnica, redes multisectoriales, indicadores de innovación.

\section{$\underline{\text { Abstract }}$}

The main goal of this paper is to analyze the relationships between cities and processes of productive innovation centered around information and communication technologies (ICT). Which are the possible methodologies to evaluate and measure innovation? This paper describes the theoretical frameworks on the indicator systems that intend to measure the production of innovations in enterprises, Universities, local governments, civil society organizations, and specifically, on the interrelations among these agents. The research uses systems of innovation measurement according to national and international indicators, and proposes indicators to analyze, measure and compare innovation processes in urban areas. The research methodology is quantitative as well as qualitative. The case studies are three cities in the Buenos Aires Province which have universities, Technological Poles, and which develop $\mathrm{R}+\mathrm{D}+\mathrm{I}$ activities in ICT -based goods and services: La Plata, Bahía Blanca and Tandil.

\section{Key Words}

Science and Technology - Socio-technical innovation - Multistakeholder networks - Innovation indicators. 
Innovación y ciudades en la sociedad de la información. Ciencia, tecnología y políticas públicas

\section{LOS ESPACIOS DE LA INNOVACIÓN URBANA}

Este artículo está basado en los avances del proyecto de investigación del Consejo Nacional de investigaciones Científicas y Técnicas (CONICET-PIP 2013-2015) "Innovación y ciudades en Sociedad de la Información: procesos, actores y resultados en tres ciudades de la provincia de Buenos Aires", y del PICT FONCYT (2014-2016) "Desarrollo local e innovación productiva en la Sociedad de la información: redes, actores y procesos en tres ciudades", dirigidos por SusANA FinQueliEvich. El objetivo general es analizar las relaciones entre las ciudades y los procesos de innovación socio-tecnológica centrados en las tecnologías de información y comunicación (TIC), considerando la interrelación entre el tamaño de la ciudad en términos poblacionales, la presencia de actores de la innovación (universidades activas en $\mathrm{I}+\mathrm{D}$, empresas de tecnología TIC, gobiernos locales interesados por innovación en el desarrollo local y organizaciones comunitarias) y la formación y desarrollo de redes entre estos actores.

Los objetivos específicos son identificar la relación existente entre el tamaño de una ciudad y los grados de innovación socio-técnica alcanzados, mediante la aplicación de sistemas de medición de la innovación según indicadores nacionales e internacionales, y la profundización de los conocimientos sobre metodologías específicas para analizar, medir y comparar procesos de innovación en áreas urbanas. Interesa analizar, mediante el estudio de actores de la innovación y sus interrelaciones, los procesos con los cuales se construyen redes de innovación a nivel local y regional, y la transferencia de conocimientos entre los actores que permiten la formación de espacios y procesos innovativos en sectores económicos relevantes para el desarrollo regional, como los bienes y servicios basados en las TIC. En este marco, se realizan estudios de caso en tres ciudades de diferente tamaño en la provincia de Buenos Aires: La Plata (649.613 habitantes), Bahía Blanca (301.531 habitantes) y Tandil (101.010 habitantes). Las tres cuentan con universidades relevantes y con polos tecnológicos, que desarrollan actividades de $\mathrm{I}+\mathrm{D}$ en bienes y servicios de base TIC.

Si bien la metodología utilizada es esencialmente cualitativa, en el proceso de trabajo surge un interrogante: ¿cómo evaluar y medir la innovación? En este artículo se plantean algunos de los conceptos sobre los indicadores que intentan medir la producción de innovaciones en empresas, universidades, gobiernos locales y organizaciones de la sociedad civil y, fundamentalmente, las innovaciones que surgen de las interrelaciones entre estos actores sociales. 


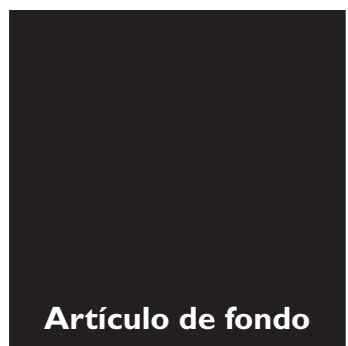

Susana Finquelievich y Patricio Feldman

Este texto describe tanto los avances empíricos, teóricos y metodológicos realizados hasta el momento, como de los interrogantes y desafíos que dichos avances han ido presentando en el curso de la investigación. Hasta el momento, hemos recogido los resultados de las primeras etapas del trabajo: relevo de información documental, realización de entrevistas a informantes clave en La Plata, Tandil y Bahía Blanca, análisis de sitios Web y portales de municipalidades, universidades nacionales radicadas en cada ciudad, polos tecnológicos, empresas, cooperativas y organizaciones de la sociedad civil.

La primera parte del artículo se refiere a la construcción de indicadores cuanti-cualitativos para evaluar el grado de desarrollo de la innovación en las tres ciudades. La segunda parte del texto se refiere a las iniciativas relevadas en diversas áreas: la existencia de gobiernos inteligentes que promuevan la participación social; la presencia de múltiples actores sociales que intervienen en la producción y difusión de productos y servicios TIC; la participación de universidades que promuevan la investigación no solo en TIC, sino también en otras áreas sociales de interés y los esfuerzos locales para incluir a los ciudadanos en los procesos de innovación productiva.

Steven Johnson (2010), al referirse a los espacios de la innovación, plantea que el tamaño de las ciudades, en número de habitantes, tiene relación directa con la generación de innovaciones. Este criterio se basa en los hallazgos del físico Geoffrey West, del Santa Fe Institute (2007, citado por JoHnson, 2010), quien ha generado un modelo que manifiesta que la manera en que las ciudades han sido construidas proviene de los patrones de la biología: mientras mayor es la ciudad, más rápidamente se generan en ella las ideas innovadoras (Finquelievich, Feldman y Fischnaller, 2013). Para Johnson (2010) las leyes de poder de WeST sugieren que una metrópolis con una población de 5 millones de habitantes es tres veces más creativa que una ciudad de 100.000 habitantes. Una de las razones es que a mayor número de gente concentrada en un territorio, existe mayor número de conexiones posibles que puedan ser formadas en el grupo y, por consiguiente, mayores posibilidades de derrame de ideas. Las buenas ideas podían encontrar su camino hacia otros cerebros y ser desarrolladas en ellos. Los economistas definen a estos flujos como "information spillover" o derrame de información. Efectivamente, la humanidad aceleró el ritmo de producción de innovaciones a partir del período situado entre los años 10.000 a. de C. y 2000 a. de C., cuando las ciudades comenzaron a construirse y se desarrollaron (JOHNSON, 2010). 
Innovación y ciudades en la sociedad de la información. Ciencia, tecnología y políticas públicas

Uno de los requisitos para la producción de innovaciones es, además de la circulación de ideas e información, su conservación como conocimiento, para poder construir sobre esa masa crítica conocimientos mayores y más innovadores. En las redes de relación de aquellas ciudades, las ideas comenzaron a difundirse, y a través de esta difusión fueron conservadas, por vía oral, escrita, por medio de imágenes, para las futuras generaciones. Esto no significa que, concentrados masivamente, los habitantes urbanos se volvieran más inteligentes o más creativos. Simplemente, se aumentaba el número de personas que pudieran producir nuevas ideas o aprovechar aquellas que estaban en circulación. No se trata aquí, como expresa JoHnson, de la sabiduría de las masas, sino de la inteligencia de alguien en la masa, que podía difundirse, concretarse y generar más y mejores innovaciones en las redes urbanas.

PierRe Lévy (2004) afirma que el desarrollo creciente de colaboración y de alianzas es un testimonio manifiesto de la importancia de las redes de innovación. Nuevas competencias deben ser importadas, producidas, instaladas permanentemente (en tiempo real) en todos los sectores. Las organizaciones deben abrirse a una circulación continua y permanentemente renovada de conocimientos científicos, técnicos, sociales o incluso estéticos. El conocimiento, según LÉvY, se ha convertido en la nueva infraestructura.

\section{QUÉ ES INNOVACIÓN, HOY}

De acuerdo con la definición incluida en la tercera edición del Manual de Oslo (OCDE, 2005), innovación es la introducción en el mercado de un producto o proceso significativamente mejorado, o bien el desarrollo de nuevas técnicas organizacionales y de marketing. Como recuerdan SCHIAVO ET ALL. (2013), las innovaciones, analizadas desde una perspectiva económica, son validadas exclusivamente por el mercado. En cambio, en el nuevo sistema de innovación que posibilitan las TIC, su validación, si bien no excluye al mercado, implica a otros sectores de la sociedad, como son el espacio científico-tecnológico, el gubernamental y la propia sociedad civil. SCHIAVO ET ÁL. plantean que la innovación consiste en la transformación de una idea en producto, servicio o proceso, nuevo o mejorado, cuya aplicación o introducción en el mercado genera valor adicional. Este valor puede ser económico o socio-ambiental, y se da en diferentes casos. Puede tratarse de la introducción de un producto nuevo o de un cambio cualitativo en un producto existente. Otra forma de innovación puede darse mediante un cambio de procesos en la industria, a través de 


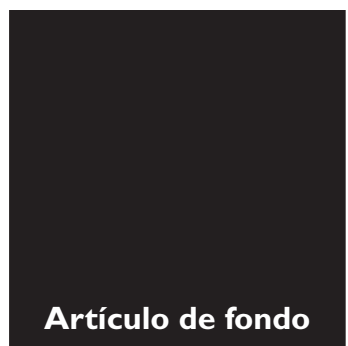

Susana Finquelievich y Patricio Feldman

la implementación de nuevos métodos organizacionales tanto en la producción como en la práctica del negocio. La apertura de un nuevo mercado en el que un área específica de la industria aún no haya penetrado también es una forma de implementar la innovación, tanto como el desarrollo de nuevas fuentes de provisión para materias primas u otras contribuciones.

En este trabajo, este concepto se amplía: se considera innovación al proceso que lleva a la difusión de un nuevo producto o procedimiento (ya sea este tecnológico, organizacional o de difusión) o a su mejora significativa, así como a su apropiación por diversos actores sociales (FINQUELIEVICH, 2012).

Respecto del análisis de los indicadores de innovación, Albornoz (2009: 19) plantea un interrogante esencial: ¿sirve la traslación directa de las categorías usadas en el mundo desarrollado para ser aplicadas a los países de América Latina? AlboRNOZ sostiene que no toda innovación es positiva. Existen innovaciones que acarrean costos sociales no aceptables para sociedades como las latinoamericanas, caracterizadas por una fuerte polarización socioeconómica e inequidad social. En sociedades sin pleno empleo, algunas innovaciones pueden ocasionar la pérdida del empleo de algunos sectores sociales, que no siempre puede ser absorbida por los nuevos conocimientos generados por el proceso innovador. No es deseable trasladar automáticamente los mecanismos que han sido exitosos en Europa o América del Norte a la realidad latinoamericana, puesto que no se trata del mismo contexto social, político, cultural, económico.

La brecha entre los países desarrollados y los que se encuentran en vías de desarrollo respecto de la innovación y el acceso al conocimiento resulta cada vez es más amplia, lo que exige a los países ubicados en el segunda categoría redoblar esfuerzos para no quedar al margen de los avances tecnológicos y científicos que operan a escala mundial. Como señala AlBORNOZ (2009: 20) en referencia al rol que debe cumplir la región, "las políticas de innovación deben ser redefinidas y enriquecidas, no sólo para aprovechar al máximo las oportunidades que ofrece el cambio tecnológico, sino para impulsar un estilo de conducta innovadora que haga posible evitar la exclusión social y ayude a atenuar la brecha de riqueza entre paises y grupos sociales".

Para medir la innovación en América Latina es preciso utilizar indicadores asociados al contexto regional, pero que permitan efectuar una comparación con el resto del mundo. Esa tensión entre originalidad y comparabilidad permitió a la OEI, junto a COLCIENCIA 
Innovación y ciudades en la sociedad de la información. Ciencia, tecnología y políticas públicas

y un grupo de expertos, elaborar el Manual de Bogotá en el año 2000. Este manual pone en discusión la utilización del Manual de Oslo (1992), pensado para las economías desarrolladas, el cual si bien constituye un aporte central para analizar los procesos de innovación a escala regional, responde a un contexto enteramente distinto del latinoamericano. El Manual de Bogotá incorpora elementos específicos del contexto regional, aunque mantiene en términos metodológicos los mismos parámetros que el Manual de Oslo, centrados en la innovación en sentido puro.

Los indicadores de innovación en América Latina deben incorporar no solo al sector industrial, sino también al sector primario y de servicios. Por ejemplo, en Argentina una gran cantidad de innovaciones emergieron en el sector agropecuario. Además, en sociedades con altos índices de exclusión social y desigualdad, resulta importante considerar las tecnologías sociales. La construcción de indicadores apropiados para medir la innovación en la región deben considerar estos aspectos. Siguiendo esta línea, AlBornoz (2009) sostiene: "es necesario construir instrumentos para recoger y procesar datos de acuerdo con conceptos teóricos adecuados para formular políticas de desarrollo científico y tecnológico enraizadas en la realidad social y que impliquen el diseño de caminos propios hacia el desarrollo".

Schiavo ET ÁL. (2013) parten de la base de que es necesario reconocer la existencia de distintas generaciones de TIC, y que ellas traen consigo problemas inéditos que interpelan a la ciencia y a la tecnología, lo cual, a su vez, tiene como consecuencia el surgimiento de conocimientos y de instituciones científicas y tecnológicas de nuevo tipo que se encuentran en distintos procesos de transformación y consolidación.

La evolución de las TIC ha contribuido a hacer revisar las actuales condiciones de desarrollo y a las variables que intervienen en él: se le atribuye un papel de gran importancia a los condicionantes sociales, como cultura, educación, historia, demografía, capital social y otros. La innovación se percibe en la actualidad no solo como un proceso económico, sino también como un fenómeno social influido por una multiplicidad de relaciones entre diversos factores sociales. El concepto "Innovación para el Desarrollo", referido a ciudades y regiones en la Sociedad de la Información o Sociedad del Conocimiento, es actualmente una de las preocupaciones prioritarias de un número creciente de países. En la última década, se han multiplicado en América del Norte, Europa y América Latina las investigaciones destinadas a analizar el rol de la innovación basada en el conocimiento en el desarrollo local (FINQUELIEVICH, 2012). 


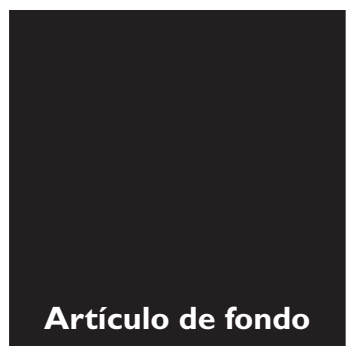

Susana Finquelievich y Patricio Feldman

Casas (2001) describe cómo Christopher Freeman, Nathan Rosemberg y otros (1991) han demostrado que la evidencia histórica cuestiona o descarta la aplicación generalizada de los modelos lineales de innovación, ya que la diferencia de tiempo entre los avances científicos y sus aplicaciones tecnológicas puede variar solo en unos meses, o bien la innovación tecnológica puede preceder al descubrimiento; en ocasiones, los avances científicos pueden estar basados en la invención de nuevas maquinarias y no en el sentido contrario.

Los procesos que dan origen a las innovaciones son extremadamente complejos y están relacionados con la emergencia y difusión de conocimientos, así como con su materialización en nuevos productos y procesos de producción (EDQUIST, 1997: 1, citado por CASAS, 2001). Uno de los primeros investigadores en analizar este tema fue MANUEL CASTELLS, quien ha estudiado el rol de las ciudades en la "nueva economía", centrada a partir de la transformación tecnológica y organizativa desarrollada desde la segunda mitad del siglo XX. En un artículo de 2007, CASTELLS analiza el vínculo entre nueva economía y política urbana como una relación complementaria: la nueva economía, facilitada por una infraestructura productiva de nuevo tipo localizada en ciertos territorios, que proporciona recursos materiales y económicos para una nueva política de calidad de vida. Esta se convierte en un factor esencial para el desarrollo de la productividad sobre el que se basa la nueva economía: una relación sinérgica.

Por su parte, Michael Gurstein (2003) plantea que el modelo de innovación, si bien está inevitablemente ligado a los proyectos nacionales, provinciales y locales, no es necesariamente un modelo originado por el Estado central "desde arriba hacia abajo", sino que puede ser considerado como basado en las comunidades locales, en su red de actores sociales, o como construido desde abajo hacia arriba.

\section{LOS INDICADORES DE LA INNOVACIÓN}

\section{La evaluación de la innovación}

Numerosos autores (Romero, Rébori y CAmio, 2010; Schiavo et Ál., 2013), retomando a BARANGER (1992), señalan que la cuantificación no es una condición necesaria para poder hablar de medición, puesto que la medida es cualquier procedimiento empírico que suponga la asignación de símbolos. Según BARANGER (1992), la construcción de un índice que exprese determinado grado de innovación puede verse como un proceso en 
Innovación y ciudades en la sociedad de la información. Ciencia, tecnología y políticas públicas

el cual se realizan operaciones para traducir un concepto en una variable que pueda ser "medible" de alguna manera (proceso de operacionalización). Comprende tres etapas, la última de las cuales es la definición operacional del concepto. Esta incluye selección de los indicadores que se ocupen de medir los aspectos que "componen" la variable general (cada indicador se asocia a una pregunta o ítem); combinación de los indicadores seleccionados en alguna clase de índice (lo cual incluye la definición del procedimiento que se llevará a cabo).

Según Romero, RéBORI y CAMIO (2010), para poder identificar el grado o nivel de innovación de las empresas, es necesario definir de qué se está hablando cuando se hace referencia a "innovación". Dicho concepto puede verse como un proceso que facilita la competitividad empresarial y contribuye al desarrollo económico de las sociedades (LunDVALL \& MASKell, 2000, citados por Romero, RÉBORI y CAMIO, 2010). La capacidad innovativa de las PyMEs, puede ser entendida como la potencialidad de idear, planear y realizar innovaciones a partir del uso de los conocimientos tecnológicos y organizativos formales e informales en la firma. Así, el proceso de innovación que se genera es resultado de la interacción entre las competencias desarrolladas, el aprendizaje que se va desarrollando, la cultura organizacional y el ambiente en el que la firma actúa (Yoguel \& BosQuerini, 1996). Sobre estos conceptos, la innovación se concibe como un proceso complejo de transformación del conocimiento genérico en conocimiento específico, a partir de las competencias desarrolladas por la firma, mediante un aprendizaje que asume riesgos idiosincrásicos y que está modelado por las características de la cultura organizacional.

De acuerdo con estos autores, no existen reglas precisas ni invariables que determinen cuál es la cantidad óptima de dimensiones para operacionalizar conceptualmente un "índice de innovación", ni tampoco la cantidad de indicadores. Cada investigación deberá seleccionar la cantidad de indicadores que considere pertinente. El proceso de construcción de este índice deja entrever que no basta solo con tener un amplio conocimiento de cuestiones teóricas y metodológicas; requiere, además, la aplicación creativa de adecuadas metodologías combinadas con cuestiones referidas al contexto y a los objetivos del estudio.

El conjunto de autores mencionado (Romero, RéBori y CAMIO, 2010) propone el INI (Índice del Nivel de Innovación), el cual crearon ad hoc para "medir" el nivel de innovación en un grupo de empresas innovadoras o potencialmente innovadoras que sean intensivas en el uso de tecnología. El proceso de construcción develó la necesidad de tomar decisiones referentes a las dimensiones que considerar, la cantidad de indicadores, la articulación 


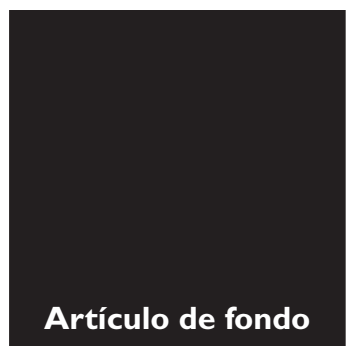

Susana Finquelievich y Patricio Feldman

de los valores (dependiendo del tipo de variables, de las unidades de medida), la función para utilizar en la combinación y las ponderaciones de los diferentes indicadores. En el proceso de identificar las dimensiones que analizar, estos autores utilizaron el Manual de Oslo (OCDE y Eurostat, 2005) y las adaptaciones realizadas para Latinoamérica y el Caribe en el denominado Manual de Bogotá (JARAMILlo ET ÁL., 2001). En estos materiales se plantea relevar cuestiones tales como el nivel de desempeño económico, las actividades de innovación (a través de indicadores de gasto y frecuencia), los resultados, objetivos, las fuentes de información y el financiamiento de la innovación. Una vez determinadas las dimensiones, se identifican las variables que actuarán como indicadores para cada una de ellas, entendiendo por indicador una característica o respuesta manifiesta que es observable.

Los indicadores que utilizaron en cada una de las dimensiones, para el caso de las pequeñas y medianas empresas (Romero, RÉBORI Y CAMIO: 2010), son los siguientes: 1) nivel de desempeño económico: participación (valor en las ventas totales de productos innovados en los últimos cinco años); 2) actividades de innovación: realización de diferentes actividades innovativas (I\&D, diseño, adquisición de tecnología y otras, las que fueron jerarquizadas y ponderadas según su importancia); asignación de recursos a cada una de estas actividades; existencia de departamentos de I\&D y de otras actividades de innovación; nivel de calificación de las personas involucradas en proyectos de I\&D y tiempo; porcentaje de inversión de la empresa de tipo interno y a través de contratos externos; 3) resultados de la innovación; introducción de productos nuevos al mercado y tipo de mercado (internacional, nacional, o solo para la empresa); en caso de existir innovación se tomó en consideración si afecta las características principales del producto, si es central al proceso y el impacto (positivo, neutro o negativo en aspectos como la rentabilidad, el flujo de caja, la participación en el mercado, entre otros), por la introducción de innovaciones de procesos, producto $u$ organizacionales en los últimos cinco años; solicitud u obtención de patentes, licencia de tecnología, posesión de procesos y productos certificados.

Según AlBORNOZ (2009), las primeras definiciones metodologías respecto de la innovación estaban orientadas más hacia la medición de resultados u "outputs" que de actividades o procesos. Con el tiempo se fueron incorporando las actividades como dimensión de análisis.

La investigación se ha nutrido de variadas herramientas metodológicas, tanto cuali- como cuantitativas - entre ellas, los mencionados indicadores, construidos por colegas-, y ha debido también relevar datos propios de manera específica y a los efectos de los objetivos 
Innovación y ciudades en la sociedad de la información. Ciencia, tecnología y políticas públicas

y los contextos de investigación elegidos. Mientras la "información” emerge en todo momento en el curso de la investigación, resulta necesario reconocer ciertos elementos como "datos", es decir, identificarlos, construirlos como tales y utilizarlos en pos de los intereses de investigación.

Los indicadores que han resultado de utilidad en este trabajo fueron aquellos que expresan la interacción multiactoral de los procesos innovativos. Hemos considerado como indicadores de utilidad, además, aquellos que remiten a la transferencia de conocimientos entre diversos actores y a los impactos de las acciones de innovación sobre el territorio local y sus dimensiones: redes sociales, economía local, medioambiente, población.

Según Rosalba Casas (2001), es posible elegir dos tipos de indicadores: los vinculados con la competitividad y crecimiento económico e indicadores de "innovación social", vinculados con políticas de inclusión social. Una segunda opción es escoger indicadores para cada uno de los actores (empresas, gobierno, sociedad civil y universidades) que se apliquen a cada ciudad. Finalmente, también se ha considerado el impacto territorial al que hemos hecho referencia.

Cuadro 1. Indicadores

\begin{tabular}{|l|l|}
\hline \multicolumn{2}{|l|}{ INDICADORES } \\
\hline Tipo de innovación \\
\hline $\begin{array}{l}\text { Competitividad y cre- } \\
\text { cimiento económico }\end{array}$ & $\begin{array}{l}\text { - Número de patentes } \\
\text { - Cantidad de investigadores (ámbito público y privado) } \\
\text { - Cantidad de graduados de universidades públicas y privadas en el territorio } \\
\text { - Número de proyectos de investigación en I + D } \\
\text { - Inversión pública en I + D } \\
\text { - Cantidad de empresas vinculadas con la CyT (pymes, grandes empresas y mul- } \\
\text { tinacionales con oficina en la ciudad) } \\
\text { - Instalación de parques o polos científico-tecnológicos y sus dimensiones: cantidad } \\
\text { de puestos de trabajo, dimensiones espaciales, cantidad de horas de actividad, etc. } \\
\text { - Número de bienes y servicios TIC de producción local }\end{array}$ \\
\hline
\end{tabular}




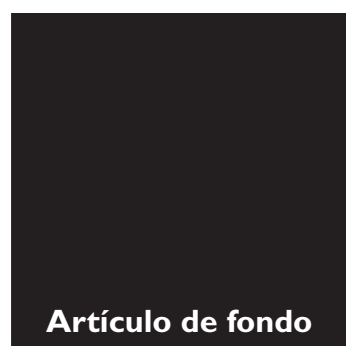

Susana Finquelievich y Patricio Feldman

\begin{tabular}{|c|c|}
\hline Innovación social & $\begin{array}{l}\text { - Proyectos de investigación vinculados con la economía solidaria, desarrollo } \\
\text { - Postenible, preservación del medio ambiente, desarrollo endógeno, etc. } \\
\text { - } \text { duales, etc. (de empresas, gobierno local y ONG) } \\
\text { - Participación de diversos actores sociales en la elaboración de políticas públicas } \\
\text { de CyT (en la etapa de diseño o en la etapa de implementación) } \\
\text { - Establecimiento de redes y alianzas entre actores de la innovación } \\
\text { - Participación activa de la sociedad civil en los proyectos y políticas públicas de } \\
\text { CyT (consultas, talleres, conferencias y diversas formas de participación) } \\
\text { - Grado de articulación con otras políticas: sociales, educativas, comerciales, } \\
\text { industriales, de salud, etc. } \\
\text { - Articulación entre lo local, regional, provincial y nacional } \\
\text { - Proyectos de voluntariado desarrollado por las universidades que incluyan } \\
\text { proyectos relacionados con la CyT (formación en programación destinada a } \\
\text { poblaciones vulnerables de la ciudad, talleres o seminarios sobre programas o } \\
\text { aplicaciones puntuales para la formación laboral, etc.) }\end{array}$ \\
\hline \multicolumn{2}{|l|}{ Actores } \\
\hline Empresas & $\begin{array}{l}\text { - Número de patentes producidas } \\
\text { - Cantidad de investigadores } \\
\text { - Número de proyectos de investigación en I + D } \\
\text { - Número de bienes y servicios TIC de producción local } \\
\text { - Interacción entre empresas de polos tecnológicos y empresas externas a ellos }\end{array}$ \\
\hline Gobierno & $\begin{array}{l}\text { - Inversión pública en I + D (\% del gasto público municipal) } \\
\text { - Indicadores de innovación en el área de gobierno electrónico }\end{array}$ \\
\hline Universidades & $\begin{array}{l}\text { - Cantidad de graduados de universidades públicas en el territorio en carreras } \\
\text { afines a la CyT } \\
\text { - Cantidad de proyectos de extensión universitaria vinculados con la CyT } \\
\text { - Cantidad de investigadores } \\
\text { - Número de licencias } \\
\text { - Transferencia de conocimientos con empresas }\end{array}$ \\
\hline Sociedad civil & $\begin{array}{l}\text { - Proyectos desarrollados por ONG u organizaciones sociales vinculados con la } \\
\text { inclusión digital o innovación social } \\
\text { - Participación en proyectos de la universidad, empresas o gobierno vinculados } \\
\text { con la innovación en bienes y servicios TIC } \\
\text { - Impactos de iniciativas innovadoras gubernamentales y empresarias sobre la } \\
\text { población (percepción) }\end{array}$ \\
\hline
\end{tabular}


Innovación y ciudades en la sociedad de la información. Ciencia, tecnología y políticas públicas

\begin{tabular}{|l|l|}
\hline \multicolumn{2}{|l|}{ Impactos sobre el territorio } \\
\hline & - Impacto del desarrollo ID local en otros lugares/escalas: intercambios o trans- \\
& ferencia de conocimientos, bienes o personal hacia otros centros de desarrollo. \\
- Concentración territorial del conocimiento: formación de investigadores/trabaja- & dores externos; publicaciones de papers; organización de eventos científicos, etc. \\
- Nuevas infraestructuras (vialidad, conectividad, equipamientos urbanos, etc.) & - Vínculo con políticas de inclusión \\
- Promoción del desarrollo local
\end{tabular}

Fuente: elaboración propia

Si bien estos indicadores no han sido aún aplicados cuantitativamente, se han empleado los criterios cualitativos para analizar los siguientes puntos: iniciativas innovadoras en gobiernos locales, formación de ciudadanía abierta a la innovación, innovación productiva, relación entre gobiernos y ciudadanía por medio de TIC. Los indicadores cuantitativos se utilizarán en la siguiente fase del proyecto.

\section{GOBIERNOS INTELIGENTES PARA CIUDADES INNOVADORAS}

Para impulsar procesos de innovación social y productiva perdurables y con solidez para impactar en el desarrollo económico y social de la población, se requieren gobiernos locales comprometidos con la innovación y el desarrollo local. Para desarrollar ciudades innovadoras se requieren gobiernos inteligentes. Estos son más que gobiernos que ofrecen servicios TIC en términos de mejorar la transparencia y la administración pública, favorecer instancias de gobierno abierto utilizando las redes sociales para comunicarse con los ciudadanos, publicando la declaración jurada de los funcionarios o permitiendo que a través del sitio Web de la municipalidad los vecinos puedan pagar sus impuestos y acceder a información pública de utilidad. Además, y fundamentalmente, impulsan espacios y entornos de innovación en las ciudades, abiertos a la participación de la ciudadanía en una perspectiva de co-creación de la innovación y el conocimiento.

Los gobiernos inteligentes se ocupan de la formación de ciudadanos para que puedan participar activamente en la cadena de innovación socio-productiva, ciudadanos que no son solo clientes o consumidores de tecnología y conocimiento, sino también productores. El término "prosumidores" sintetiza esta dualidad, en la que los ciudadanos consumen pero 


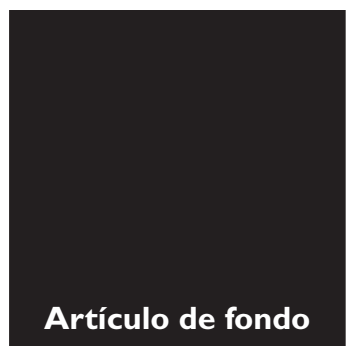

Susana Finquelievich y Patricio Feldman

también producen información y conocimiento, e intervienen en innovaciones tecnológicas y sociales. Un ejemplo es la creación por parte del gobierno municipal de Bahía Blanca de la Agencia de Gobierno Abierto e Innovación en el año 2011. Esta agencia, que tiene el rango de secretaría y trabaja en conjunto con las otras áreas de gobierno (desarrollo social, economía, salud, educación, etc.), tiene como objetivo principal "Diseñar, proponer y coordinar las políticas de innovación municipal en las distintas secretarías y direcciones del gobierno municipal, investigando y analizando experiencias internacionales de innovación gubernamental y proyectos de ciudades inteligentes a fin de evaluar su incorporación". La agencia asume el desafío de "Proponer, diseñar e implementar proyectos de gobierno abierto con el objetivo de transparentar información municipal, promover la participación y la colaboración con los diferentes actores de la sociedad civil'.

La Municipalidad de La Plata dispone de una Secretaría de Presupuesto Participativo que desarrolla el programa de presupuesto participativo, cuyo principal objetivo es "(...) que los propios ciudadanos; primero reunidos en asambleas barriales y luego en Consulta Popular propongan y decidan la prioridad de las obras públicas para ser realizadas en cada una de sus localidades (...)". Si bien los proyectos son publicados en sitio Web de la municipalidad, el gobierno local no utiliza todavía los medios digitales para implementar y desarrollar esta política, de forma tal que se pueda incrementar el flujo de participación e interacción entre los ciudadanos y el gobierno.

Ninguna ciudad puede llevar a cabo un proceso de innovación abierta sin la participación activa del gobierno local. Ningún gobierno puede llamarse inteligente si no estimula la participación pública en el desarrollo de la innovación socio-productiva.

\section{LA RELACIÓN ENTRE LOS GOBIERNOS LOCALES Y LOS CIUDADANOS A TRAVÉS DE LAS TIC}

Una de las características de una ciudad innovadora se refiere a la relación fluida y transparente entre el gobierno local y los ciudadanos, a través del uso de las TIC. La municipalidad de Bahía Blanca cuenta con un portal de gobierno abierto, donde los ciudadanos pueden realizar preguntas a los funcionarios y proponer iniciativas al intendente. En la sección de datos abiertos, los ciudadanos acceden a las declaraciones juradas de los funcionarios, el presupuesto municipal, información acerca del intendente, programas sociales, etc. El gobierno se comunica con los ciudadanos a través de las redes sociales, como Twitter y Facebook. 
Innovación y ciudades en la sociedad de la información. Ciencia, tecnología y políticas públicas

El gobierno de la ciudad de La Plata utiliza la telefonía móvil para comunicarse con los ciudadanos, a partir de dos iniciativas: a) envía a través de SMS información referida a la disponibilidad de lugares para estacionar; $b$ ) informa a los ciudadanos, también vía SMS, en caso de alerta meteorológica. Estas iniciativas sirven como ejemplo de políticas públicas que atienden un contexto específico e intentan resolver problemáticas locales: el tráfico y las inundaciones. La municipalidad de Tandil no tiene un portal de gobierno abierto y no utiliza las redes sociales para comunicarse con los ciudadanos, si bien estos pueden enviar por correo electrónico sugerencias, reclamos e inquietudes al intendente de la ciudad.

La falta de herramientas de interacción entre gobiernos y ciudadanos, evidencia, muchas veces, la falta de herramientas que estimulen la participación pública en los procesos de innovación productiva. Por eso, de la mano de la creación de gobiernos inteligentes, se forman ciudadanos activos e innovadores.

De los tres casos estudiados, Bahía Blanca es la ciudad que más ha avanzado en fortalecer y crear vínculos entre gobierno y ciudadanos a partir del uso de las TIC. El Portal de Gobierno Abierto y la Agencia de Gobierno Abierto e Innovación son un reflejo de este proceso. El gobierno actual presentó en 2014 un proyecto de ordenanza para que el Gobierno Abierto sea obligatorio para las gestiones de gobierno futuras, con el objetivo de sostener en el tiempo las políticas públicas tendientes a la modernización de la administración pública y el acercamiento entre los ciudadanos y el gobierno local.

\section{¿SE PUEDE FORMAR CIUDADANOS INTELIGENTES?}

La creación de polos tecnológicos en las ciudades de La Plata, Tandil y Bahía Blanca constituye un ejemplo concreto de aplicación de políticas transversales y multisectoriales, en las que participan múltiples actores sociales (fundamentalmente universidades, empresas y gobiernos locales) en el proceso de innovación productiva. Sin embargo, una ciudad no se define como innovadora solo por contar con un polo tecnológico. Es necesario formar a los ciudadanos para que puedan participar en el diseño e implementación de innovaciones socio-productivas y tecnológicas. No basta con generar entornos o espacios para la innovación: se requiere también formar y capacitar a los ciudadanos en habilidades TIC, emprendedorismo, e-democracia, entre otros conocimientos que favorecen la innovación y el desarrollo local. ¿Qué se puede hacer sobre este tema desde las políticas públicas? 


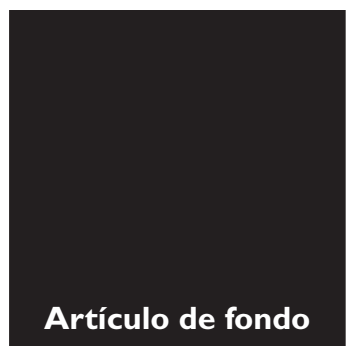

Susana Finquelievich y Patricio Feldman

Las ciudades innovadoras y los gobiernos inteligentes necesitan impulsar instancias como seminarios, Workshops, Hackatons, ferias de innovación, City Camps, museos interactivos, exposiciones de ciencia y tecnología, entre otros, abiertos a la participación de los científicos y ciudadanos. Además, la presencia de universidades y centros de investigación que promueven actividades de I+D permite formar los recursos humanos que necesitan las empresas locales y el polo tecnológico para generar procesos de innovación a escala local.

En el caso de la ciudad de Bahía Blanca, la Agencia de Gobierno Abierto e Innovación organizó el primer Hackaton con el objetivo de "desarrollar modelos de comunicación claros para la Sociedad en temas de Medio Ambiente, Salud y Desarrollo Social, sin excluir otros temas de interés que puedan surgir en el evento, así como también promover el desarrollo de aplicaciones que brinden información útil a los ciudadanía o instituciones". Participaron del evento numerosos actores sociales: funcionarios de la Municipalidad, programadores independientes, investigadores, periodistas, académicos, expertos en Salud, Desarrollo Social y Medio Ambiente y representantes de ONG. Como resultado, los programadores participantes diseñaron un prototipo de plataforma de comunicación en temas referidos a la Salud y el Medio Ambiente y han desarrollado un sistema inteligente de transporte de información que funciona a través de SMS y permite a los ciudadanos recibir información precisa sobre los horarios del transporte público en tiempo real. Esto es un ejemplo de cómo los ciudadanos pueden generar conocimientos y tecnologías para mejorar la calidad de vida y bienestar de la sociedad.

Además, el gobierno de Bahía Blanca ha desplegado en algunos barrios postergados los Centros de Alfabetización Digital (CAD), con el fin de capacitar y alfabetizar a niños y adultos en el manejo de las TIC: "Educación con TIC no es solamente el uso instrumental de las nuevas tecnologías, sino que implica el aprendizaje de competencias de gestión de información, comunicación, intercambio con otros en un mundo global y capacidad de actualización permanente. Hay una intención de entender a estas tecnologías como un recurso psico-social fundamental para la comunidad".

En la ciudad de La Plata el gobierno local organizó en el año 2012 la feria TEC, una exhibición de la producción local en ciencia y tecnología. Participaron empresas, universidades nacionales e instituciones públicas, en una muestra de trabajo en red entre el gobierno local y otros actores sociales para difundir la producción e innovación en TIC. Ahora bien, este evento no se ha repetido. 
Innovación y ciudades en la sociedad de la información. Ciencia, tecnología y políticas públicas

Acercar la ciencia a la sociedad, estimular la participación pública en materia de políticas de innovación y desarrollo local, construir redes entre los actores de la innovación (universidades, empresas, gobierno, organizaciones de la sociedad civil, etc.) permite distribuir geográfica y socialmente el conocimiento y el desarrollo económico y hacer que las ciudades sean polos de desarrollo tecnológico y no meras receptoras de las innovaciones.

\section{LAS CIUDADES Y LA INNOVACIÓN PRODUCTIVA}

Los gobiernos locales de los casos estudiados han tomado medidas e impulsado políticas específicas, en diferentes grados y en distintas áreas, para promover la innovación productiva. Los procesos de innovación no están desligados del contexto económico y social, y operan en aquellos sectores de la economía que son más dinámicos en un momento histórico determinado. La incorporación de tecnología y conocimiento al proceso productivo constituye una fuente para agregar valor a la cadena de producción, como en el caso de las actividades económicas tradicionales como la agricultura, específicamente el sector de las oleaginosas, en el que el aporte realizado por la biotecnología ha permitido generar mayor valor agregado.

El caso de la ciudad de Tandil es emblemático. Su economía está basada principalmente en la agricultura, la ganadería, el turismo y la actividad minera. También se han instalado en la ciudad, entre otras, varias empresas de desarrollo de software, a medida que crece su reciente polo tecnológico. La creación del Parque Científico Tecnológico en la Universidad del Centro de la Provincia de Buenos Aires (UNICEN) es una iniciativa de articulación público/privada en torno a la incorporación de tecnología a la producción agrícola/ganadera. Se han firmado alrededor de sesenta acuerdos entre empresas nacionales e internacionales, asociaciones de productores, el gobierno local y la UNICEN, en materia de investigaciones y desarrollo en áreas como salud y producción agrícola. La universidad aporta el soporte científico en temas relativos a Toxicología, Metabolismo, Psicología y Genética, lo que permite mejorar la producción agropecuaria, pero también estimular la investigación y desarrollo para agregarle valor y conocimiento.

En el polo están presentes empresas del sector que buscan establecer un polo de desarrollo de software que actúe a nivel local y regional. Más de veinte empresas nacionales e internacionales se encuentran participando del PI. La idea es transferir los aportes del campo 


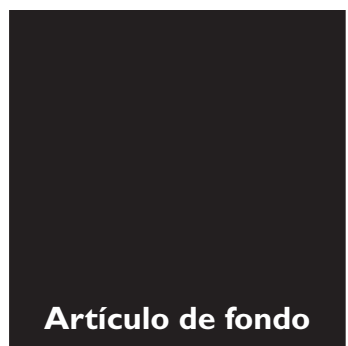

Susana Finquelievich y Patricio Feldman

informático a la actividad económica central de la ciudad, la agricultura y ganadería, de modo que un sector económico pujante sirva como motor de desarrollo de otro sector con gran perspectiva de futuro.

La incorporación de las TIC al proceso productivo no solo se limita a las actividades económicas predominantes en algunas ciudades de la provincia, como la agricultura y la ganadería. La presencia de universidades, polos tecnológicos y pymes permite impulsar actividades como el desarrollo de software y servicios informáticos, que implican un cambio con respecto a las condiciones naturales e históricas de estas ciudades. Un ejemplo de esta complementación es el proyecto de "Educación Continua" impulsado por la Escuela de Veterinaria de la UNICEN, que se propone diseñar un prototipo de software para almacenar, generar y centralizar información sobre la producción de carne bovina. El software ayudaría a las empresas a optimizar el proceso de producción, a partir de contar con mejores indicadores, registros y almacenamiento de datos.

La existencia de un puerto comercial de gran envergadura transformó a Bahía Blanca en un polo de comercio importante para la provincia de Buenos Aires. Se trata del mayor centro petroquímico del país: concentra el $66 \%$ de la producción nacional en este rubro. La creación del polo tecnológico y la instalación de empresas dedicadas al desarrollo de software permitieron iniciar un camino complementario de desarrollo local, priorizando la incorporación de las TIC de forma integral. En el año 2013 se inauguró el Centro Nacional de Micro y Nanotecnología del Bicentenario, cuyo objetivo central es "promover la investigación y el desarrollo sustentable de la industria en Micro y Nano electrónica a nivel nacional, a través de la participación de empresas productoras de bienes y servicios $y$ del sector académico, propiciando la sustitución de importaciones del sector y generando los mecanismos tendientes a facilitar la participación de la pymes".

Con la participación del Instituto Nacional de Tecnología Industrial (INTI), la Universidad Nacional del Sur (UNS), la Universidad de San Martín (UNSAM), la Universidad Tecnológica Nacional (UTN), el gobierno de Bahía Blanca, empresas como Coradir, Unitec Blue, Probatery, Microtro, La Asociación de Fábricas Argentinas Terminales de Electrónica (AFARTE) y La Cámara Argentina de Industrias Electrónicas, Electromecánicas y Luminotécnicas (CADIEL), el centro prevé la producción de circuitos integrados de alta complejidad, utilizables en telefonía celular, televisores inteligentes y computadoras, entre otros dispositivos. 
Innovación y ciudades en la sociedad de la información. Ciencia, tecnología y políticas públicas

En el año 2009 la ciudad de La Plata inauguró el Parque Tecnológico para promover la industria TIC con el fin de estimular la innovación y desarrollo. Este polo fue planeado para competir con los de Rosario y Córdoba, dos de los más grandes a nivel nacional en el rubro de la industria tecnológica. El Distrito Informático del Gran La Plata (DILP), un clúster de empresas nacionales e internacionales, forma parte del parque, junto al gobierno local y la Universidad Nacional de La Plata (UNLP), una de las más importantes del país en términos de formación de recursos humanos para la industria. El objetivo principal es transformar a la ciudad en un "Silicon Valley propio".

En La Plata también se localizan la Universidad Católica de La Plata, la Universidad Notarial Argentina, la Universidad del Este. Cuenta también con una Facultad Regional de la Universidad Tecnológica Nacional y con la Universidad Pedagógica. Estas atraen a estudiantes de todo el país y del extranjero, dándole a la ciudad una rica vida cultural joven, permeable a las innovaciones.

\section{CONCLUSIONES}

El análisis de los procesos de innovación productiva en entornos urbanos y la conformación de redes de innovación a escala local constituyen modos de abordar la investigación sobre las problemáticas del desarrollo local en un mundo globalizado. El creciente avance tecnológico y el peso específico de la $\mathrm{C} \& \mathrm{~T}+\mathrm{i}$ en el aparato productivo estimulan a los gobiernos locales, a las empresas y a las universidades a tomar medidas para impulsar la incorporación del conocimiento a la producción. Las ciudades cobran mayor relevancia y se configuran como entornos favorecedores de la innovación.

En definitiva, la ciudad es un gran tubo de ensayo, un laboratorio viviente. El mero hecho de experimentar hace mover las ruedas del desarrollo. Tal como señala Levy (2004), es importante constituir colectivos inteligentes que partan de una idea básica: "la inteligencia está repartida en todas partes". La inteligencia colectiva no puede desprenderse del contexto cultural en el que se desarrolla. Está en todas partes, es global, pero anclada a una realidad local que le da sentido y sustento. No se trata de un concepto abstracto, sino que se remite a las relaciones sociales.

Es necesario generar sistemas locales que puedan integrar los conocimientos con las realidades locales. El carácter relacional de la inteligencia colectiva implica pensar cómo 


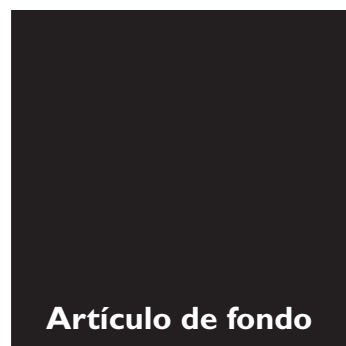

Susana Finquelievich y Patricio Feldman

generar redes de innovación en las ciudades que logren potenciar el vínculo entre los actores de la innovación y las necesidades de la comunidad en la que se inscriben. No es el desarrollo económico el que "empodera" a la sociedad, sino el empoderamiento de la sociedad el que impulsa el desarrollo económico.

Por otro lado, el tamaño de las ciudades influye en la generación de innovaciones, precisamente por el potencial para crear redes de conocimiento entre los diversos actores sociales. Si la inteligencia está en todas partes, la posibilidad de vínculo es más importante cuanto mayor sea el tamaño de la población. En ciudades grandes, las ideas fluyen y circulan con mayor velocidad, las posibilidades de que "lleguen a buen puerto" son mayores, dado el alcance y la probabilidad de acertar en el blanco. Las relaciones sociales constituyen el pilar dinámico de la economía del conocimiento: cuanto mayor sea el potencial de vínculo, mayor será la probabilidad de que surjan nuevas ideas.

Por último, ha sido interesante notar en los últimos años que el avance de los procesos de innovación comienza a fundamentarse no solo en los productos innovadores, sino también en los procesos de producción de estos. Si bien tanto los desarrollos de software como de tecnologías aplicadas se han basado siempre en la identificación de necesidades sociales o económicas, se ha constituido en los últimos años un nuevo desafío para empresas, universidades y comunidades científicas, que consiste precisamente en lograr que el plus innovador de sus resultados sea haber cambiado la manera como se produce y conoce tradicionalmente, a partir de involucrar en los procesos creativos a comunidades y grupos sociales específicos. Estos grupos pueden abandonar así la mera lógica de la necesidad y el consumo para tomar parte en la lógica de producción y creación de conocimiento científico e innovación.

El grado en que estos procesos se están desarrollando en el país y en los territorios abordados en este trabajo es aún poco relevante en relación con el gran desarrollo que están teniendo en los países más industrializados. Sin embargo, la tendencia mundial de fomentar este tipo de procesos comienza a vislumbrarse, en particular en las ciudades de mayor potencial innovador.

Según se puede deducir del grado de avance de nuestra investigación, el tamaño de una ciudad condiciona el grado de innovación socio-productiva, pero no inhibe ni garantiza por sí mismo la posibilidad de avanzar en procesos innovativos. El rol de los gobiernos locales y del Estado en el diseño e implementación de políticas públicas de desarrollo científico- 
$\underline{\text { Innovación y ciudades en la sociedad de la información. Ciencia, tecnología y políticas públicas }}$

tecnológico y productivo resulta esencial para generar innovación local en ciudades, al menos en las ciudades grandes y medianas.

La inteligencia colectiva se construye de manera colaborativa, y habilita la constitución de ciudades y ciudadanos inteligentes e innovadores.

\section{BIBLIOGRAFÍA}

ALBORNOZ, M. (2009). "Indicadores de innovación: las dificultades de un concepto en evolución". En: revista CTS. N. ${ }^{\circ} 13$, vol. 5, noviembre de 2009 http://www.redalyc.org/ pdf/924/92415269002.pdf.

BARANGER, D. (1992) Construcción y análisis de datos. Una introducción al uso de técnicas cuantitativas en la investigación social. Editora Universitaria de Misiones, Posadas. CASAS, Rosalba, comp. (2001) La formación de redes de conocimiento. Una perspectiva regional desde México. Instituto de Investigaciones Sociales de la UNAM/Anthropos, Barcelona.

CASTELLS, Manuel (2007). "Science and Technology in Society: from Biotechnology to the Internet" [Reseña del libro del mismo título de Daniel Lee Kleinman]. Contemporary Sociology, Vol. 36, No. 2, march 2007.

FERREIRA DE BARROS, Fernando Antonio (2005). "A tendencia concentradora da producao do conhecimento no mundo contemporáneo". Paralelo 15 - COPPE - ABITPI, Rio de Janeiro.

FINQUELIEVICH, Susana (2012). "Innovación en los territorios de la sociedad del conocimiento". ANPUR (Associação Nacional de Pós-Graduação e Pesquisa em Planejamento Urbano e Regional), XIV Encontro Nacional, Quem planeja o território? Atores, arenas e estratégias, Rio de Janeiro, de 23 a 27 de mayo de 2011. http://xivenanpur.com.br/ FINQUELIEVICH, Susana y FELDMAN, Patricio (2013). "Apropiación social de las TIC, innovación y resistencia”. Ponencia presentada al Foro Internacional Apropiación Social de TIC para la planeación participativa del desarrollo. Universidad Javeriana, Bogotá, Colombia.

FINQUELIEVICH, Susana (2013). "Innovación abierta en las ciudades: las nuevas formas de participación por medio de TIC", Quito Digital 2013. XIV Encuentro Iberoamericano de Ciudades Digitales, Quito, 25 y 26 de septiembre de 2013. 


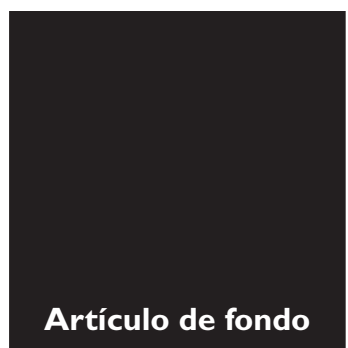

Susana Finquelievich y Patricio Feldman

GURSTEIN, Michael. "Effective Use: A Community Informatics Strategy Beyond the Digital Divide", First Monday, december 2003, http://ojphi.org/htbin/cgiwrap/bin/ojs/ index.php/fm/article/viewArticle/1107.

JARAMILLO, H.; LUGONES, G. y SALAZAR, M. (2001) Normalización de indicadores de innovación tecnológica en América Latina y el Caribe: manual de Bogotá. RICYT/ OEA/CYTED COLCIENCIAS/ OCYT.

JOHNSON, Steven (2010). "Where good ideas come from. The natural history of innovation". Penguin Books, New York.

LEVY, Pierre (2004). "Inteligencia colectiva. Por una antropología del ciberespacio". BIREME, OPS, OMS, Washington DC, www.inteligenciacolectiva.bvsalud.org.

LUNDVALL, Bengt Ake, editor (2010). "National Systems of Innovation. Towards a Theory of Innovation and Interactive Learning". Anthem Press, Londres.

OCDE \& EUROSTAT (2005) Manual de Oslo: Guía para la Recogida e Interpretación de Datos sobre Innovación. European Communities: OECF.

OECD. Oslo Manual: Guidelines for Collecting and Interpreting Innovation. Data, 3rd Edition. http://www.oecd.org/document/33/0,3343,en_2649_34451_35595607_1_1_1_1,00. html

RÉBORI, A.; CAMIO, M. y ROMERO, M. (2010). "Un índice para 'medir' el nivel de innovación tecnológica en empresas intensivas". En RAI, Revista de Administração e Inovação. Vol. 7, núm. 1, enero-marzo, 2010. Universidade de São Paulo, Brasil, http:// www.redalyc.org/articulo.oa?id=97317009002.

RÉBORI, A.; CAMIO, M. y ROMERO, M. (2007). "Tipologías culturales de empresas innovadoras: un estudio en empresas del parque científico tecnológico de la UNICEN, Argentina". En: Anais do Seminario Latino-Iberoamericano de Gestión Tecnológica, 12, Buenos Aires, Argentina.

SCHIAVO, Ester; BAUMANN, Pablo; DOS SANTOS NOGUEIRA, Camilla y VERA, Paula (2013). Estudio sobre indicadores TIC en instituciones científicas y tecnológicas de Iberoamérica. OEI - AECID, Buenos Aires, http://www.observatoriocts.org/files/Archivo\%20Documental/Documentos\%20de\%20proyectos/ticoctsREVISADO.pdf.

WEST, Geoffrey B. et ál. (2009). "Power Laws in Urban Supply Networks, Social Systems, and Dense Pedestrian Crowds". En: Complexity Perspectives in Innovation and Social Change Methodos Series, 2009, Vol. 7, Part IV, 433-450, DOI: 10.1007/978-14020-9663-1_17. 